\title{
Retrospective analysis of the diagnostic yield of newborn drug testing
}

\author{
Kelly E Wood ${ }^{1 *}$, Lori L Sinclair ${ }^{2}$, Carolyn D Rysgaard ${ }^{2}$, Frederick G Strathmann ${ }^{3,4}$, Gwendolyn A McMillin ${ }^{3,4}$ \\ and Matthew D Krasowski
}

\begin{abstract}
Background: The objective of this study was to identify high-yield screening risk factors for detecting maternal non-medical drug use during pregnancy.

Methods: A four year retrospective analysis was conducted at an academic medical center. Detailed chart review of both the newborn and mother's medical record was performed on all cases for which one or more drug(s) or metabolite(s) were identified and confirmed in meconium or urine.

Results: 229 (9.2\%) of 2,497 meconium samples out of 7,749 live births confirmed positive for one or more non-medical drugs. History of maternal non-medical drug and/or tobacco use in pregnancy was present in $90.8 \%$ of non-medical drug use cases. Addition of social risk factors and inadequate prenatal care increased the yield to 96.9\%.
\end{abstract}

Conclusions: Use of focused screening criteria based on specific maternal and social risk factors may detect many prenatal non-medical drug exposures.

Keywords: Meconium drug abuse detection/testing, Urine drug abuse detection/testing, Non-medical drug use, Fetal drug exposure, Prenatal drug abuse, Substance abuse testing

\section{Background}

Reported non-medical drug use among pregnant women in the United States is estimated at a rate of 5 per 100 births based on most recent national survey data [1]. This is likely an underestimate due to known underreporting by pregnant women $[2,3]$.

The lack of universal clinical indications for newborn drug testing in the United States results in variable screening practices [4]. For most institutions, universal screening of newborns for maternal non-medical drug use is impractical and not cost effective [5,6]. Legally, providers are required to notify child protective services when a drug-exposed infant or child is identified per the Child Abuse Prevention and Treatment Act [7].

Analysis of meconium to detect fetal drug exposure has traditionally been the gold standard for newborn drug screening. Because meconium production starts

\footnotetext{
* Correspondence: kelly-wood@uiowa.edu

'Stead Family Department of Pediatrics, University of lowa Children's Hospital, lowa City, IA 52242, USA

Full list of author information is available at the end of the article
}

around the 12th week of gestation, analysis can theoretically detect second and third trimester drug use. Disadvantages of meconium drug testing include detection of medications administered to the newborn prior to meconium collection, inconsistent distribution of analytes in the heterogeneous meconium matrix, and missed collections $[4,8]$.

Urine drug testing is widely used in newborn drug testing but has a short detection window capturing maternal non-medical drug use up to 3 to 7 days prior to delivery depending on the half-life of the drug $[4,9]$. Dilute urine or delayed collection may result in a false negative screen even in the setting of recent maternal drug use. Like meconium, urine drug testing in newborns may pick up medications given to newborn prior to sample collection $[4,9]$.

Multiple maternal risk factors are associated with nonmedical drug use during pregnancy which often involves multiple substances including ethanol and illicit drugs [2,10-12]. Prenatal drug abuse may increase a mother's risk of premature delivery, placental abruption, and 
precipitous delivery while also contributing to low birth weight and intrauterine growth restriction $[5,13]$. Other than ethanol, data are conflicting regarding the association between maternal non-medical drug abuse with congenital malformations $[5,14]$.

Protocols for identifying which newborns to screen present a number of challenges for the clinical and social work team. Some of the highest risk factors for maternal non-medical drug use - such as maternal history of drug abuse, previous child protective services involvement, or domestic violence - may be difficult to elicit from the parent(s), especially if a mother presents to a facility that did not handle her prenatal care. When using meconium as the specimen for newborn drug testing, there is a risk that collection may be missed if risk factors emerge days after delivery. Thus, narrow criteria for newborn drug testing runs some risk of missing cases. On the other hand, overly broad criteria may increase sensitivity at the expense of specificity.

The objective of this study was to review newborn urine and meconium drug screening to identify high-yield screening risk factors to detect maternal non-medical drug use during pregnancy. The newborn drug testing protocol at the study institution has been used at many facilities in the state of Iowa [15].

\section{Methods}

\section{Retrospective analysis at academic medical center}

Retrospective analysis was conducted of the medical records of all newborns who had urine and/or meconium drug analysis studies performed over a four year period $(6 / 2 / 2008-5 / 31 / 2012 ; n=2,851)$ at the University of Iowa Hospitals and Clinics (UIHC). This retrospective study was Institutional Review Board approved. UIHC is a state academic medical center that serves as a tertiary care center. The medical center includes high-risk obstetric services and a level IV neonatal intensive care unit. By institutional practice, the decision to perform newborn drug screening is based on assessment of 29 items related to maternal, delivery, and newborn risk factors (see Additional file 1). The assessment tool was implemented in 2007 (prior to period of retrospective study in this report) and used throughout the entire period of retrospective analysis.

Detailed chart review was performed on all cases $(\mathrm{n}=581)$ for which one or more $\operatorname{drug}(\mathrm{s})$ or metabolite(s) were identified and confirmed in meconium, and also on a randomly selected subset of 200 of the 1,916 cases (across all 4 years) without any drugs identified in meconium (random selection used "Random Sample of Cases" in SPSS, PASW Statistics 18, Chicago, IL, USA). For newborns that had only urine and not meconium drug testing performed $(n=354)$, all cases with positive results were reviewed in detail. Chart review included birth history (gestational age, birth weight, delivery complications), maternal history, indications for newborn drug screening, identification of prescribed medications for mother and newborn, demographics, and health insurance (summarized in Table 1). Results of meconium drug analyses performed by a national reference laboratory (ARUP Laboratories, Salt Lake City, UT, USA) over the same four year time period $(6 / 1 / 2008-5 / 31 / 2012)$ were used for comparison.

As detailed in Figure 1 and below in the Results section, four groups were defined based on drug(s) and/or drug metabolite(s) detected by meconium drug analysis: 1. Group A-cases where testing was negative; 2. Group B-cases explained by prescribed medications given to mother and/or newborn; 3. Group C-cases where non-medical drug use was detected; 4. Group D-cases not explained by medications prescribed for mother or newborn. Categorization of drugs detected in Group C is summarized in Table 2. Group $\mathrm{D}$ is described in more detail in the Results section.

\section{Drug testing analysis}

Meconium samples were analyzed by a reference laboratory (ARUP Laboratories) using enzyme-linked immunosorbent assay (ELISA) with confirmation by gas chromatography/ mass spectrometry (GC/MS) or liquid chromatography tandem mass spectrometry (LC/MS/MS) testing [16,17]. Results were only reported if the ELISA and confirmation methods agreed, eliminating likelihood of false positives.

Urine drug testing was performed using homogeneous immunoassays (Roche Diagnostics, Indianapolis, IN, USA) performed in the UIHC clinical laboratory. All confirmatory urine drug testing was referred to a reference laboratory (ARUP Laboratories) for analysis and quantitation by GC/MS or LC/MS/MS.

The Additional file 1 contains detailed description of analytical methods and the perinatal risk assessment tool used at the institution of study.

\section{Statistical analysis}

Statistical analyses were carried out in SPSS. Maternal age, gestational age, gravida (number of times a woman has been pregnant), para (number of pregnancies carried to viable gestational age), and days meconium collected after birth are summarized as median and interquartile range. All other data were expressed as frequencies. The differences between Group C (non-medical drug use) and the other groups were tested by Fisher's exact method.

\section{Results}

\section{Overall rates of drug testing}

During the period of study at UIHC, 7,749 live births occurred. Drug testing - urine or meconium or both was ordered in $36.8 \%$ of live births $(n=2,851)$. The success rate of collecting meconium and urine was $87.6 \%(n=2,497)$ and $74.6 \%(n=1,773)$ respectively. 
Table 1 Demographics, birth statistics, and health insurance status

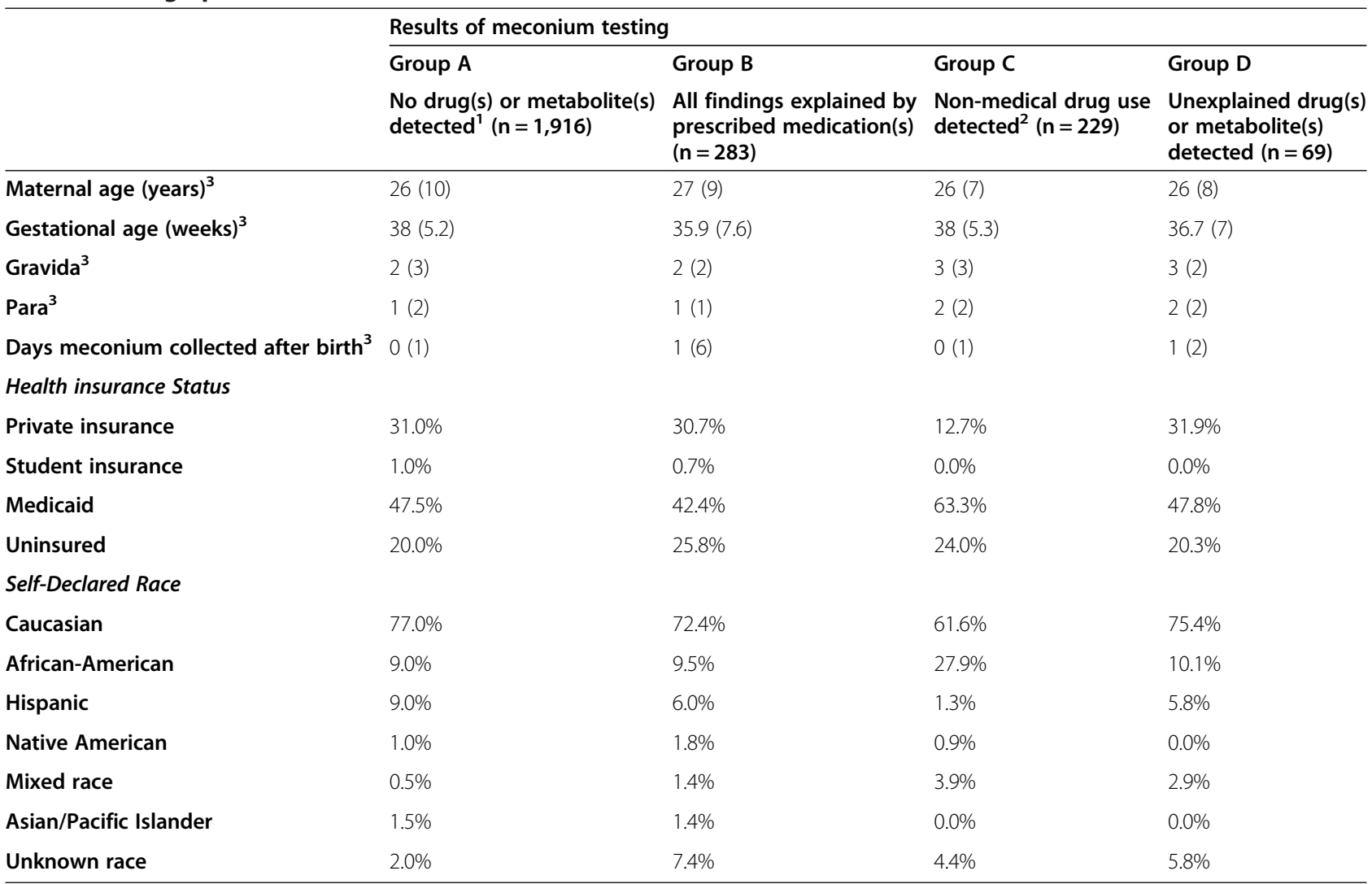

Random sample of 200 within the total group of 1,916 was reviewed in detail and used for frequency calculations.

${ }^{2}$ Non-medical drug use includes amphetamines (amphetamine, methamphetamine, Ecstasy), benzodiazepines, cannabis, cocaine, and opioids used outside of health professional outpatient prescription or inpatient administration.

${ }^{3}$ Data for these variables are presented as median (interquartile range). Gravida is the number of times a woman has been pregnant. Para is the number of pregnancies carried to viable gestational age.

Detection of non-medical drug use was reported to child protective services. Figure 1 outlines the results of testing in the study population.

Table 1 provides demographic, birth data, and health insurance status for the study populations using the four groups defined in Methods and in Figure 1.

\section{Meconium testing results}

Of the 2,497 cases with meconium analysis, 1,916 (76.7\%) were entirely negative for drug(s) and/or drug metabolite(s) (Group A). One or more drug(s) and/or drug metabolite(s) was detected in $581(23.3 \%)$ of meconium samples.

There were 283 (11.3\%) newborns for whom the compounds detected in meconium could be explained by prescribed medications given to mother and/or newborn (Group B). The most common of these medications included morphine $(\mathrm{n}=165,58.3 \%)$, lorazepam $(\mathrm{n}=86$, $30.4 \%$ ), codeine with or without presumed metabolites such as morphine or hydrocodone $(\mathrm{n}=41,14.5 \%)$, and phenobarbital $(n=22,7.8 \%)$. All positive tests for lorazepam and phenobarbital were explained by administration of prescribed medication to the newborn prior to meconium collection. Of the 165 positive tests for morphine in the absence of codeine, 157 occurred in newborns administered morphine prior to meconium collection; 8 were explained by maternal prescription for morphine, typically administration in the perinatal period. A variety of other drug(s) and/or drug metabolite(s) were detected that were consistent with documented maternal outpatient prescriptions in the 2nd and 3rd trimester including amphetamine $(\mathrm{n}=4,1.4 \%)$, butalbital $(\mathrm{n}=3,1.1 \%)$, methadone $(\mathrm{n}=4,1.4 \%)$, nordiazepam (a metabolite of diazepam and chlordiazepoxide; $\mathrm{n}=2$, $0.7 \%)$, and propoxyphene ( $\mathrm{n}=1,0.4 \%)$.

Non-medical drug use was detected in 229 (9.2\%) of meconium samples analyzed (Group C). Tetrahydrocannabinol (THC) $(\mathrm{n}=172,75.1 \%)$ was the most common drug detected. Within this group, opioids detected included codeine ( $\mathrm{n}=19,8.3 \%)$, hydrocodone alone $(\mathrm{n}=7,3.1 \%)$, hydrocodone and oxycodone together $(n=6,2.6 \%)$, morphine alone $(n=3$, $1.3 \%)$, oxycodone alone $(\mathrm{n}=1,0.4 \%)$, oxycodone and codeine together $(n=1,0.4 \%)$, and propoxyphene $(n=3$, $1.3 \%)$. Other non-medical drugs detected include amphetamines, benzodiazepines, and cocaine (Table 2). 


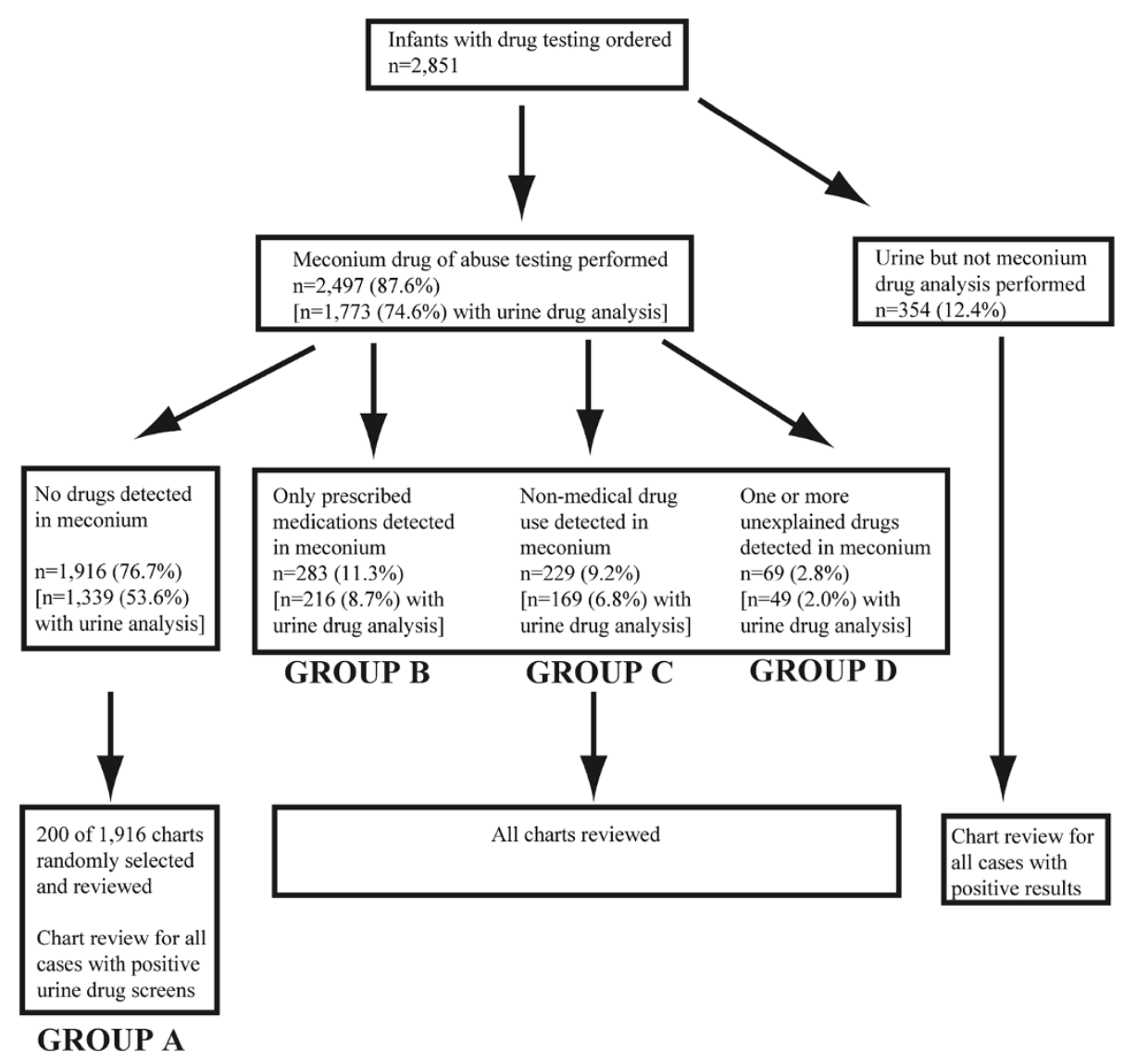

Figure 1 Flow diagram of the study population categorized by results of meconium and urine drug testing. The designation of Groups A, B, C, and $\mathbf{D}$ are described in detail in the Methods section.

There were an additional 69 (2.8\%) newborns who had $\operatorname{drug}(\mathrm{s})$ and/or drug metabolite(s) detected that were not explained by medications prescribed for mother or newborn (Group D); however, there was insufficient evidence to demonstrate non-medical drug use. In some cases, incomplete access to maternal medication history (often due to patients whose primary medical care was in other cities or even out of state) prevented full examination of prescription medication history. The majority of these cases $(n=58,84 \%)$ involved various combinations of codeine with or without other opiates. The remainder were other opiates $(n=10,14.4 \%)$ or diazepam $(\mathrm{n}=1,0.01 \%)$. Without clear evidence of non-medical drug use, no child protective report was pursued.

\section{Comparison to Meconium Drug Analysis Results from National Reference Laboratory}

Figure $2 \mathrm{~B}$ shows meconium testing results over a 4 year period from a de-identified database from a national reference laboratory (ARUP Laboratories). Phencyclidine was detected in $0.1 \%$ of ARUP specimens but none of the Iowa specimens. The higher rate of positivity of barbiturates and benzodiazepines in the Iowa dataset was primarily accounted for by phenobarbital $(0.9 \%$ in Iowa vs. $0.6 \%$ at ARUP) and lorazepam (3.4\% in Iowa vs. $0.5 \%$ at ARUP). Given that the national reference laboratory dataset is de-identified, it is unknown what proportion of results is non-medical drug use versus prescribed medications.

\section{Findings in urine}

Of the 2,497 newborns for whom meconium specimens were analyzed, $1,773(71.0 \%)$ had concurrent urine testing. Only twelve urine samples $(0.7 \%)$ screened positive for non-medical drug use ( 8 THC, 2 cocaine, 2 methamphetamine). Nine of those samples were confirmed positive by GC/MS or LC/MS/MS. The other three urine samples screened positive for THC metabolite but negative by confirmation. All twelve samples positive by screening (whether confirmed or not) demonstrated $100 \%$ concordance with meconium testing. In 128 cases where meconium analysis detected non-medical drug use, a concurrent urine specimen was negative for the $\operatorname{drug}(\mathrm{s})$ detected in meconium. There were four 
Table 2 Categorization of non-medical drug use found in meconium

\begin{tabular}{|c|c|}
\hline Findings in meconium ${ }^{1}$ & $\begin{array}{l}\text { Number of } \\
\text { newborns }\end{array}$ \\
\hline \multicolumn{2}{|l|}{ AMPHETAMINES ( $n=10,4.4 \%)$} \\
\hline Amphetamine & 1 \\
\hline Amphetamine and hydrocodone & 1 \\
\hline Methamphetamine $+/-$ amphetamine ${ }^{2}$ & 6 \\
\hline Methamphetamine and $\mathrm{THC}^{2}$ & 2 \\
\hline \multicolumn{2}{|l|}{ BENZODIAZEPINES $(n=3,1.3 \%)$} \\
\hline Alprazolam & 1 \\
\hline Nordiazepam & 1 \\
\hline Temazepam & 1 \\
\hline \multicolumn{2}{|l|}{ COCAINE $(n=16,7 \%)$} \\
\hline Cocaine & 11 \\
\hline Cocaine and THC & 5 \\
\hline \multicolumn{2}{|l|}{ OPIOIDS ( $n=40,17.5 \%)$} \\
\hline Codeine $+/$ - metabolites & 14 \\
\hline Codeine and THC & 5 \\
\hline Hydrocodone $+/$ - metabolites & 7 \\
\hline Hydrocodone and oxycodone & 6 \\
\hline Morphine $+/$ - metabolites & 3 \\
\hline Oxycodone & 1 \\
\hline Oxycodone and codeine & 1 \\
\hline Propoxyphene +/- norpropoxyphene & 3 \\
\hline \multicolumn{2}{|l|}{ CANNABINOIDS $(n=172,75.1 \%)$} \\
\hline THC alone & 160 \\
\hline THC and other & 12 \\
\hline TOTAL NEWBORNS WITH NON-MEDICAL DRUGS & 229 \\
\hline
\end{tabular}

urine specimens that were immunoassay screen positive for amphetamines but negative by LC/MS/MS confirmatory analysis. Labetalol administration in the mother was suspected to be the likely cause of these false positives, given the reported ability of labetalol metabolites to cause amphetamine positive screens with some amphetamines immunoassays [18].

An additional 354 newborns had urine drug testing performed in the absence of meconium testing. Of these, only 14 had one or more positive results on urine drug screening. All findings were explained by medications administered to the newborn prior to sample collection except for one newborn with positive urine immunoassay screen for THC. In this newborn, there was insufficient urine specimen for confirmatory analysis, and the child abuse report was rejected.
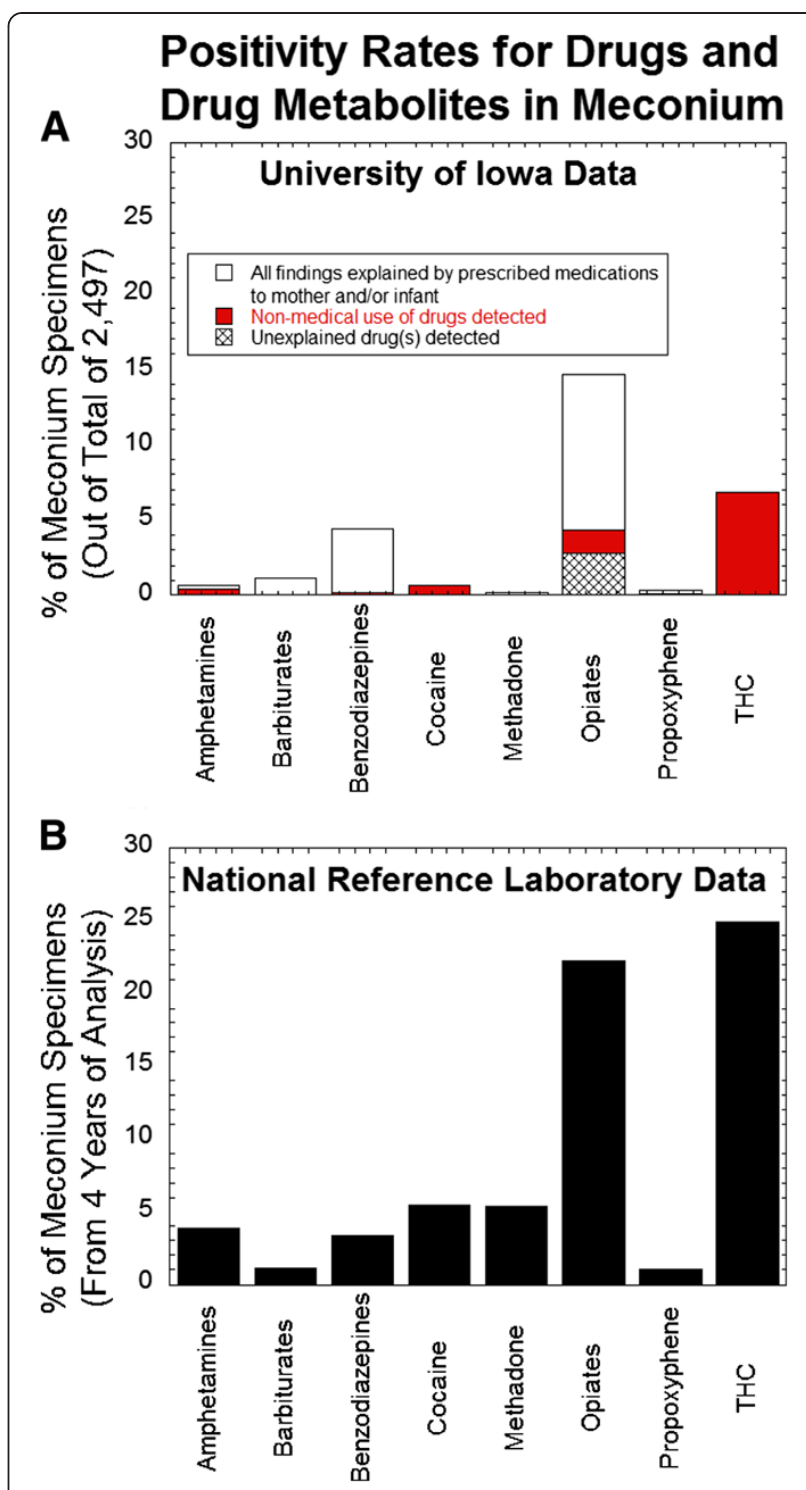

Figure 2 Positivity rates for selected drugs and drug classes in meconium. Data shown includes that observed at the University of lowa site (panel $\mathbf{A}$ ) versus de-identified data from a national reference laboratory (panel B). Percent positivity represents the number of unique positive specimens divided by the total number of specimens tested (not positivity rates for individual analytes).

\section{Yield of Screening Criteria}

We examined how well various risk factors from the assessment tool correlated with the identification of maternal non-medical drug use (Figure 3; Table 3). A history of maternal non-medical drug use, specifically unexplained positive drug screen during pregnancy or self-report of or documented prior non-medical drug use, and tobacco use during pregnancy were significantly higher in Group C compared to the other groups (Fisher's Exact Test $\mathrm{p}<0.001$ for all factors). The addition of poor or late prenatal care (Fisher's Exact Test $\mathrm{p}<0.05$ ) and/or specific social risk factors such as maternal 


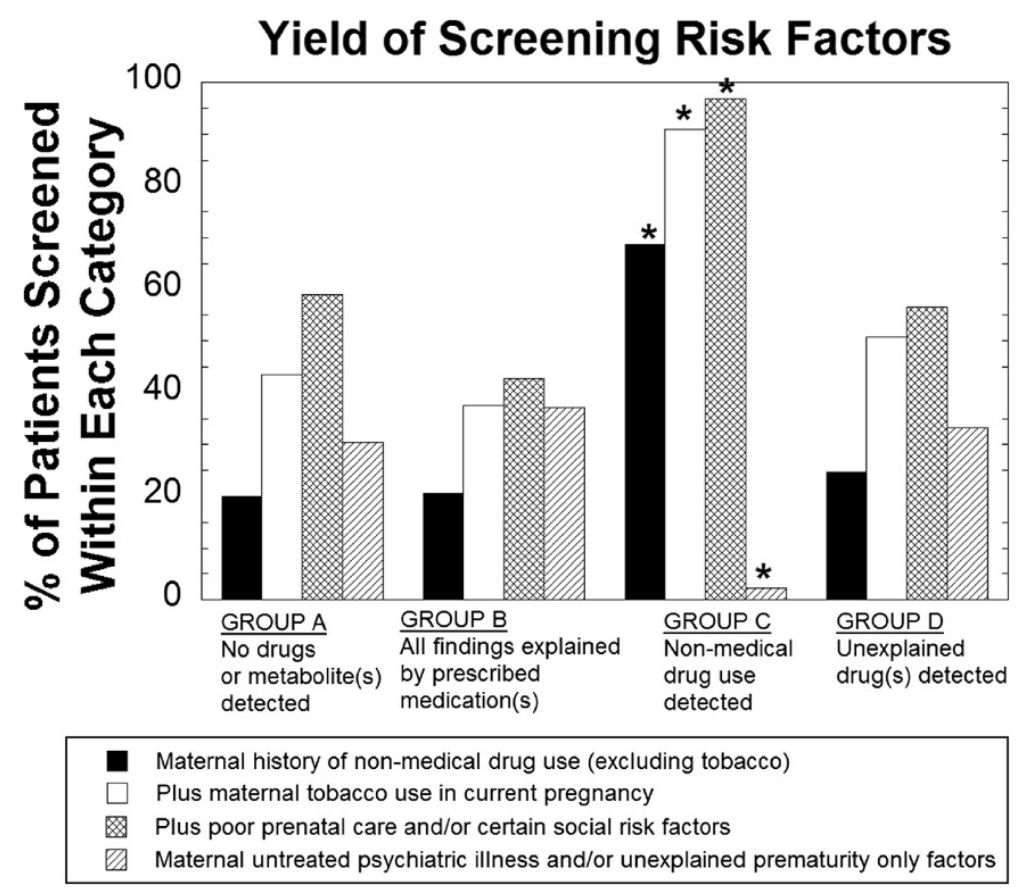

Figure 3 Yield of screening risk factors for identifying maternal non-medical drug use. History of maternal non-medical drug use, tobacco use in current pregnancy, inadequate prenatal care, and/or certain social risk factors were found in $96.9 \%$ of cases of non-medical drug use. Unexplained prematurity and/or untreated maternal psychiatric illness in the absence of other criteria were found in only $1.7 \%$ of non-medical drug use but accounted for approximately $30 \%$ of the remaining categories. ${ }^{*} p<0.005$, Fisher's Exact Test for comparison of Group C vs. Groups A, B, and D.

incarceration (Fisher's Exact Test $\mathrm{p}<0.05$ ) were present in all but 7 of the 229 cases (96.9\%) within Group C. The seven newborns (3.1\%) not meeting the above risk criteria were all cases with only THC detected. Additionally, unexplained hepatitis $B$, hepatitis $C$, or human immunodeficiency virus infection (Fisher's Exact Test $\mathrm{p}<0.05$ ) was significantly more common in Group C.

Cases for which newborn drug testing was performed solely due to the presence of either untreated maternal psychiatric illness and/or unexplained prematurity (i.e. no other risk factors present) were significantly less frequent in Group C (1.7\%) versus Group A (30.5\%), Group B (37.1\%), or Group D (26.1\%).

\section{Discussion}

In this study the prevalence of maternal non-medical drug use per meconium drug testing at the study institution during the four year study period was $3 \%$, which is below national estimates of 5\% [1]. A large de-identified database of meconium analysis from a national reference laboratory showed higher percentages of samples positive for amphetamines, phencyclidine, propoxyphene, cocaine, opiates, and THC compared to the study institution.

We found the presence of a maternal history of nonmedical drug use, tobacco use in current pregnancy, incarceration, prior child protective services involvement, domestic violence, and/or inadequate prenatal care accounted for $96.9 \%$ of cases in which non-medical drug use was detected in meconium. Maternal tobacco use combined with a history of other non-medical drug use was present in $90.8 \%$ of cases in which a non-medical drug was detected. Neither untreated maternal psychiatric illness nor unexplained prematurity in the absence of other risk factors were predictive of maternal non-medical drug use in our study. These two factors alone were the reason for screening in approximately $30 \%$ of cases.

Specific risk factors that were significantly more frequent in the cases with non-medical drug use in pregnancy included unexplained positive drug screen during pregnancy, maternal self-report of or documented prior non-medical drug use including previous pregnancies, tobacco use during current pregnancy, poor or late prenatal care, incarceration of mother, and unexplained hepatitis B, hepatitis C, and/or human immunodeficiency virus infections. It should be noted, however, that some of these risk factors were seen in a high rate of cases without drugs or drug metabolites detected in meconium. For example, within group A (no drugs or drug metabolites detected), 33.0\% had tobacco use in current pregnancy, $16.5 \%$ had poor or late prenatal care, and $59.0 \%$ fell within the broad grouping of risk factors involving non-medical drug use, poor or late prenatal care, and social risk factors. 
Table 3 Most common risk factor indications for newborn drug testing

\begin{tabular}{|c|c|c|c|c|}
\hline & \multicolumn{4}{|c|}{ Results of meconium testing } \\
\hline & Group A & Group B & Group C & Group D \\
\hline & $\begin{array}{l}\text { No } \operatorname{drug}(s) \text { or } \\
\text { metabolite(s) } \\
\text { detected }^{1} \\
(n=1,916)\end{array}$ & $\begin{array}{l}\text { All findings explained } \\
\text { by prescribed } \\
\text { medication(s) } \\
(\mathrm{n}=283)\end{array}$ & $\begin{array}{l}\text { Non-medical drug } \\
\text { use detected }{ }^{2} \\
(n=229)\end{array}$ & $\begin{array}{l}\text { Unexplained drug(s) } \\
\text { or metabolite(s) } \\
\text { detected } \\
(n=69)\end{array}$ \\
\hline \multicolumn{5}{|l|}{ History of maternal non-medical drug use ${ }^{2}$} \\
\hline Unexplained positive drug screen during pregnancy & $1.5 \%$ & $3.2 \%$ & $14.0 \% \%^{* * *}$ & $2.9 \%$ \\
\hline Maternal self-report of prior non-medical drug use & $4.5 \%$ & $4.9 \%$ & $27.1 \% * * *$ & $1.4 \%$ \\
\hline Non-medical drug use in previous pregnancy & $0.5 \%$ & $0.4 \%$ & $3.1 \%^{* *}$ & $0.0 \%$ \\
\hline Previous infant exposure to non-medical drug use & $1.0 \%$ & $0.0 \%$ & $0.9 \%$ & $0.0 \%$ \\
\hline Documented prior history of non-medical drug use & $16.0 \%$ & $15.5 \%$ & $52.4 \%^{* * *}$ & $17.4 \%$ \\
\hline Tobacco use during current pregnancy & $33.0 \%$ & $24.0 \%$ & $51.1 \% * * *$ & $36.2 \%$ \\
\hline \multicolumn{5}{|l|}{ Inadequate Prenatal care } \\
\hline Poor ( $\leq 4$ visits) or late (after week 16 ) prenatal care & $16.5 \%$ & $5.7 \%$ & $22.7 \% * *$ & $7.2 \%$ \\
\hline \multicolumn{5}{|l|}{ Maternal/Family Social risk factors } \\
\hline History of domestic violence by partner & $4.5 \%$ & $2.8 \%$ & $6.6 \%$ & $4.5 \%$ \\
\hline History of child abuse/protective services involvement & $4.0 \%$ & $3.9 \%$ & $5.2 \%$ & $2.9 \%$ \\
\hline Incarceration & $2.0 \%$ & $3.5 \%$ & $6.6 \% *$ & $1.4 \%$ \\
\hline Total maternal non-medical drug use excluding tobacco & $20.0 \%$ & $20.5 \%$ & $68.6 \% * * *$ & $24.6 \%$ \\
\hline Total maternal non-medical drug use including tobacco & $43.5 \%$ & $37.5 \%$ & $90.8 \% * * *$ & $50.7 \%$ \\
\hline Total including prenatal care and social risk factors & $59.0 \%$ & $42.8 \%$ & $96.9 \% * * *$ & $56.5 \%$ \\
\hline Untreated maternal psychiatric illness ${ }^{3}$ & $22.5 \%$ & $21.9 \%$ & $20.5 \%$ & $20.3 \%$ \\
\hline Unexplained prematurity & $26.5 \%$ & $57.6 \%$ & $22.3 \%$ & $46.4 \%$ \\
\hline Cases screened solely due to two factors above & $30.5 \%$ & $37.1 \%$ & $1.7 \% * * *$ & $26.1 \%$ \\
\hline \multicolumn{5}{|l|}{ Other factors } \\
\hline Unexplained placental abruption & $1.0 \%$ & $3.9 \%$ & $0.9 \%$ & $2.9 \%$ \\
\hline Unexplained maternal HBV, HCV, or HIV infection ${ }^{4}$ & $0.5 \%$ & $1.8 \%$ & $3.5 \%{ }^{*}$ & $1.4 \%$ \\
\hline Unexplained infant seizures, stroke, brain infarction & $0.5 \%$ & $3.9 \%$ & $0.4 \%$ & $2.9 \%$ \\
\hline Congenital malformations in newborn & $5.5 \%$ & $14.5 \%$ & $1.3 \%$ & $7.2 \%$ \\
\hline Maternal age $<18$ years old & $3.7 \%$ & $3.8 \%$ & $0.8 \%$ & $2.8 \%$ \\
\hline
\end{tabular}

${ }^{1}$ Random sample of 200 within the total group of 1,916 was reviewed in detail and used for frequency calculations.

${ }^{2}$ Non-medical drug use includes amphetamines (amphetamine, methamphetamine, Ecstasy), benzodiazepines, cannabis, cocaine, and opioids used outside of health professional outpatient prescription or inpatient administration.

${ }^{3}$ This category was for untreated maternal psychiatric illness (e.g. major depression, bipolar disorder) excluding non-medical drug use.

${ }^{4}$ Abbreviations: HBV, hepatitis B virus; HCV, hepatitis $C$ virus; HIV, human immunodeficiency virus.

*p $<0.05$, Fisher's Exact Test. Group C vs. Groups A, B, and D.

**p $<0.005$, Fisher's Exact Test. Group C vs. Groups A, B, and D.

*** $p<0.001$, Fisher's Exact Test. Group C vs. Groups A, B, and D.

Prior studies suggest marijuana is the most common non-medical drug used during pregnancy, consistent with our data in which THC accounted for $75 \%$ of non-medical drug use $[10,19]$. Although delta-9-THC, the active ingredient in marijuana, crosses the placenta, the association of prenatal marijuana use with premature delivery, low birth weight, or congenital malformations is not clear [3,5,20-23]. Long term, prenatal marijuana exposure may have adverse effects on learning, attention and behavior [5]. Three urine specimens in our study screened positive for THC but showed negative confirmatory testing, a phenomenon previously noted in other studies $[24,25]$.

Opiate exposure is estimated to occur in 2 to $20 \%$ of pregnancies [26]. Recent studies using meconium drug testing have documented misuse of prescription pain medication among pregnant women $[27,28]$. The incidence of non-medical use of prescription opiates identified via meconium drug testing was $0.5 \%(n=40)$ in this study. This figure excludes the 68 unexplained positive meconium samples for opiates. Interpretation of positive opiate screens 
is complicated given the complexity of opiate metabolism and multiple possible sources of opiates such as medications prescribed for the mother and/or newborn, poppy seeds, heroin, or intentional misuse of prescription opiates (See Additional file 1) [9].

A major finding of this study is that detection of prescribed medications is common with meconium and/or urine drug screening. The study institution includes a neonatal intensive care unit which cares for many premature infants. Many premature infants receive medications prior to the passage of meconium, which may be delayed until the ninth day of life [28]. Morphine, lorazepam, and phenobarbital administered to newborns prior to meconium collection accounted for $96.5 \%$ of the 283 samples whose meconium findings were completely explained by prescribed medications. Detection of prescribed medications requires thorough review of the maternal and newborn medical records, along with consideration of the metabolic pathways of opiates and benzodiazepines, to avoid unnecessary accusations of non-medical drug use (See Additional file 1).

Our study found very low yield of urine drug screening. Over a 4 year period, urine drug screening did not detect any non-medical drug use not seen in meconium. In addition, urine drug testing failed to detect 128 cases of non-medical drug use determined by meconium analysis.

Study limitations include analysis of a single academic site with a primarily Caucasian patient population and incomplete data for chart review for some patients. Only newborns meeting protocol criteria had screening ordered (63.2\% of the live births in the University of Iowa sample did not meet screening criteria). Some risk criteria for newborn drug screening were dependent on maternal self-reporting of data. Incarceration was not a formal screening criterion per the study institution's protocol and only identified if specifically mentioned in the clinical documentation. The protocol used was specific to the institution. False negative tests may have occurred if sample drug concentrations fell below testing cutoffs. In addition, maternal non-medical drug use may have been missed in some cases if there was both prescription and non-medical use of the same drug or drugs sharing common metabolites during pregnancy (e.g. non-medical use of morphine by mother in pregnancy but administration of prescription morphine to the newborn prior to meconium collection). Specimens were not collected on all newborns with drug testing ordered, a known challenge given the logistic challenges with performing wide scale newborn drug testing.

Future studies using umbilical cord for newborn drug testing would be of interest to compare with the findings in meconium. Unlike meconium, universal collection of umbilical cord specimens at birth is feasible. Specimens can be held upwards of 2 weeks, allowing for newborn drug testing to occur even when risk factors emerge days after birth. Use of umbilical cord would also avoid detection of newborn medications, which in our population was a very common finding.

\section{Conclusions}

Overall, our study demonstrates that maternal history of non-medical drug use and tobacco use in pregnancy were the highest yield risk factors for identifying non-medical drugs in meconium. Inadequate prenatal care and social risk factors were also helpful. Newborn urine drug testing was poor for detecting maternal non-medical drug use and has little diagnostic yield. Meconium drug testing frequently detects prescribed medications, necessitating a thorough review of the pharmacy history. Our results suggest that focused screening criteria based on specific maternal risk factors may detect many prenatal nonmedical drug exposures.

\section{Additional file}

\section{Additional file 1: Microsoft Word document with detailed} description of analytical methods, the perinatal risk assessment tool used at the institution of study, and metabolic pathways of opiates and benzodiazepines.

\section{Abbreviations}

ELISA: Enzyme-linked immunosorbent assay; GC/MS: Gas chromatography/ mass spectrometry; LC-MS/MS: Liquid chromatography tandem mass spectrometry; THC: Tetrahydrocannabinol; UIHC: University of lowa Hospital and Clinics; HBV: Hepatitis B virus; HCV: Hepatitis C virus; HIV: Human immunodeficiency virus.

\section{Competing interests}

The authors all declare that they have no competing interests.

\section{Authors' contributions}

All authors listed are responsible for the reported research in this manuscript and all have participated in the concept and design, analysis and interpretation of data, drafting or revising or the manuscript, and have approved the manuscript as submitted.

\section{Acknowledgement}

The authors would like to acknowledge Dr. Jeffrey Segar for his editorial contribution.

\section{Author details}

${ }^{1}$ Stead Family Department of Pediatrics, University of lowa Children's Hospital, lowa City, IA 52242, USA. '2Department of Pathology, University of lowa Hospitals and Clinics, lowa City, IA 52242, USA. ${ }^{3}$ ARUP Institute for Clinical and Experimental Pathology, Salt Lake City, Utah, USA. ${ }^{4}$ Department of Pathology, University of Utah School of Medicine, Salt Lake City, Utah, USA.

Received: 6 December 2013 Accepted: 8 July 2014

Published: 29 July 2014

\section{References}

1. Substance Abuse and Mental Health Services Administration: Results from the 2011 National Survey on Drug Use and Health: Summary of National Findings. In NSDUH Series H-44. Rockville, MD: Substance Abuse and Mental Health Services Administration; 2012.

2. Lester BM, EISohly M, Wright LL, Smeriglio VL, Verter J, Bauer CR, Shankaran S, Bada HS, Walls HC, Huestis MA, Finnegan LP, Maza PL: The maternal lifestyle 
study: drug use by meconium toxicology and maternal self-report. Pediatrics 2001, 107(2):309-317.

3. van Gelder MM, Reefhuis J, Caton AR, Werler MM, Druschel CM, Roeleveld $\mathrm{N}$, National Birth Defects Prevention S: Characteristics of pregnant illicit drug users and associations between cannabis use and perinatal outcome in a population-based study. Drug Alcohol Depend 2010, 109(1-3):243-247.

4. Cotten SW: Drug testing in the neonate. Clin Lab Med 2012, 32(3):449-466.

5. Behnke M, Smith VC, Committee on Substance A, Committee on F, Newborn: Prenatal substance abuse: short- and long-term effects on the exposed fetus. Pediatrics 2013, 131(3):e1009-e1024.

6. Wong S, Ordean A, Kahan M: Substance use in pregnancy. J Obstet Gynaecol Can 2011, 33(4):367-384

7. Gateway CWI: Parental Drug use as Child Abuse.. Washington, DC: U.S. Department of Health and Human Services, Children's Bureau; 2012.

8. Gray TR, Eiden RD, Leonard KE, Connors GJ, Shisler S, Huestis MA: Identifying prenatal cannabis exposure and effects of concurrent tobacco exposure on neonatal growth. Clin Chem 2010, 56(9):1442-1450.

9. Farst KJ, Valentine JL, Hall RW: Drug testing for newborn exposure to illicit substances in pregnancy: pitfalls and pearls. Int J Pediatr 2011, 2011:951616.

10. De Santis M, De Luca C, Mappa I, Quattrocchi T, Angelo L, Cesari E: Smoke, alcohol consumption and illicit drug use in an Italian population of pregnant women. Eur J Obstet Gynecol Reprod Biol 2011, 159(1):106-110.

11. McFarlane J, Parker B, Soeken K: Physical abuse, smoking, and substance use during pregnancy: prevalence, interrelationships, and effects on birth weight. J Obstet Gynecol Neonatal Nurs 1996, 25(4):313-320.

12. Schempf AH: Illicit drug use and neonatal outcomes: a critical review. Obstet Gynecol Surv 2007, 62(11):749-757.

13. Smith LM, LaGasse LL, Derauf C, Grant P, Shah R, Arria A, Huestis M, Haning W, Strauss A, Della Grotta S, Liu J, Lester BM: The infant development, environment, and lifestyle study: effects of prenatal methamphetamine exposure, polydrug exposure, and poverty on intrauterine growth. Pediatrics 2006, 118(3):1149-1156.

14. Creanga AA, Sabel JC, Ko JY, Wasserman CR, Shapiro-Mendoza CK, Taylor P, Barfield W, Cawthon L, Paulozzi L: Maternal drug use and its effect on neonates: a population-based study in Washington State. Obstet Gynecol 2012, 119(5):924-933.

15. Oral R, Strang T: Neonatal illicit drug screening practices in lowa: the impact of utilization of a structured screening protocol. J Perinatol 2006, 26(11):660-666.

16. Marin $\mathrm{SJ}$, Keith $\mathrm{L}$, Merrell M, McMillin GA: Comparison of drugs of abuse detection in meconium by EMIT II and ELISA. J Anal Toxicol 2009, 33(3):148-154.

17. Marin SJ, Merrell M, McMillin GA: Drugs of abuse detection in meconium: a comparison between ELISA and biochip microarray. J Anal Toxicol 2011, 35(1):40-45.

18. Yee LM, Wu D: False-positive amphetamine toxicology screen results in three pregnant women using labetalol. Obstet Gynecol 2011, 117(2 Pt 2):503-506.

19. Gaalema DE, Higgins ST, Pepin CS, Heil SH, Bernstein IM: Illicit drug use among pregnant women enrolled in treatment for cigarette smoking cessation. Nicotine Tob Res 2013, 15(5):987-991.

20. Bada HS, Das A, Bauer CR, Shankaran S, Lester BM, Gard CC, Wright LL, Lagasse L, Higgins R: Low birth weight and preterm births: etiologic fraction attributable to prenatal drug exposure. J Perinatol 2005, 25(10):631-637.

21. Day N, Sambamoorthi U, Taylor P, Richardson G, Robles N, Jhon Y, Scher M, Stoffer D, Cornelius M, Jasperse D: Prenatal marijuana use and neonatal outcome. Neurotoxicol Teratol 1991, 13(3):329-334.

22. Fergusson DM, Horwood LJ, Northstone K, Team AS: Maternal use of cannabis and pregnancy outcome. BJOG 2002, 109(1):21-27.

23. van Gelder MM, Reefhuis J, Caton AR, Werler MM, Druschel CM, Roeleveld $\mathrm{N}$, National Birth Defects Prevention S: Maternal periconceptional illicit drug use and the risk of congenital malformations. Epidemiology 2009, 20(1):60-66.

24. Barakauskas VE, Davis R, Krasowski MD, McMillin GA: Unresolved discrepancies between cannabinoid test results for infant urine. Clin Chem 2012, 58(9):1364-1367.
25. Cotten SW, Duncan DL, Burch EA, Seashore CJ, Hammett-Stabler CA: Unexpected interference of baby wash products with a cannabinoid (THC) immunoassay. Clin Biochem 2012, 45(9):605-609.

26. Moller M, Karaskov T, Koren G: Opioid detection in maternal and neonatal hair and meconium: characterization of an at-risk population and implications to fetal toxicology. Ther Drug Monit 2010, 32(3):318-323.

27. Buchi KF, Suarez C, Varner MW: The prevalence of prenatal opioid and other drug use in utah. Am J Perinatol 2013, 30(3):241-244.

28. Launiainen T, Nupponen I, Halmesmaki E, Ojanperä I: Meconium drug testing reveals maternal misuse of medicinal opioids among addicted mothers. Drug Test Anal 2013, 5(7):529-533.

doi:10.1186/1471-2393-14-250

Cite this article as: Wood et al:: Retrospective analysis of the diagnostic yield of newborn drug testing. BMC Pregnancy and Childbirth 2014 14:250.

\section{Submit your next manuscript to BioMed Central and take full advantage of:}

- Convenient online submission

- Thorough peer review

- No space constraints or color figure charges

- Immediate publication on acceptance

- Inclusion in PubMed, CAS, Scopus and Google Scholar

- Research which is freely available for redistribution

Submit your manuscript at www.biomedcentral.com/submit
() Biomed Central 